\title{
Wide-field 3D optical imaging using temporal focusing for holographically trapped microparticles
}

\author{
Roman Spesyvtsev ${ }^{1}{ }^{*}$, Helen A. Rendall $^{1}$, And Kishan Dholakia ${ }^{1}$ \\ ${ }^{1}$ SUPA, School of Physics and Astronomy, University of St. Andrews, North Haugh, St. Andrews, Fife KY16 9SS, Scotland \\ *Corresponding author: rs222@st-andrews.ac.uk
}

Compiled September 24, 2015

\begin{abstract}
A contemporary challenge across the natural sciences is the simultaneous optical imaging or stimulation of small numbers of cells or colloidal particles organised into arbitrary geometries. We demonstrate the use of temporal focusing with holographic optical tweezers in order to achieve depth-resolved two-photon imaging of trapped objects arranged in arbitrary three dimensional geometries using a single objective. Trapping allows independent position control of multiple objects by holographic beam shaping. Temporal focusing of ultrashort pulses provides a wide-field two-photon depthselective activation of fluorescent samples. We demonstrate wide-field depth-resolved illumination of both trapped fluorescent beads and trapped HL60 cells in suspension with full 3D positioning control. These approaches are compatible with implementation through scattering media and can be beneficial for emergent studies in colloidal science and particularly optogenetics, offering targeted photoactivation over a wide area with $\mu \mathrm{m}$ depth control precision. (๑) 2015 Optical Society of America
\end{abstract}

OCIS codes: (180.6900) Microscopy, three-dimensional microscopy; (180.2520) Microscopy, fluorescence microscopy; (140.7010) Lasers and laser optics, laser trapping.

http://dx.doi.org/10.1364/ao.XX.XXXXXX

Targeted photo-activation has been widely used to study and control cellular processes for an extended period of time. While single-photon methods have pioneered the field of optogenetics, two-photon photo-stimulation methods are gaining more popularity due to their superior penetration depth through tissue and the ability of depth selectivity [1,2]. The former is the result of the lower scattering for the longer wavelengths, which are used for two-photon excitation. The latter is due to the non-linear excitation efficiency which limits the excitation volume to places with high light density. These advantages are especially valuable for selective activation of cells in complex arrangements. The need for an extended illumination area in optogenetics makes temporal focusing (TF) of ultrashort pulses a favorable technique for two-photon activation [2-4]. This technique was originally proposed for multiphoton wide-field imaging in order to provide depth sectioning for large transverse illumination fields, removing the need for the scan in the transverse plane compared to standard multiphoton techniques. Since then, TF has been adopted to non-linear imaging, optogenetics and lithography techniques [5-10] but has not been combined particularly with other biophotonics approaches. The depth sectioning in TF is achieved by temporally stretching an illumination pulse everywhere except the image plane where the pulse is compressed $[3,4]$. The multiphoton excitation volume is, therefore, reduced to a thin layer in the imaging plane where the photon density is maximized. In terms of optogenetics, TF has been applied to two-photon fluorescence 3D imaging [11] and neural activation $[5,9]$. The depth resolution for TF is comparable to standard two-photon fluorescence techniques [11] and it can be combined with structural illumination techniques in order to enhance lateral and axial resolutions $[8,12]$. Importantly, it was recently demonstrated that TF improves the penetration depth into the scattering sample compared to standard two-photon fluorescence [9]. Together with imaging, optical micro-manipulation is also moving towards applications in such 'complex' environments. The advances in beam shaping for optical trapping have enabled trapping in scattering and turbulent media with in situ aberration corrections [13]. Optical trapping has been successfully used for manipulation of microparticles and cells in vitro [14-16] and most recently in vivo [17]. Furthermore, many imaging techniques have been combined with optical tweezers and successfully applied to tomographic microscopy and 3D imaging [18-22] though these have almost exclusively focused upon single photon or holographic approaches. The use of wide field multiphoton imaging in this regard may have significant advantages.

In this work we present a combination of temporal focusing with holographic optical tweezers allowing wide-field depthresolved multiphoton illumination of trapped objects in arbitrary arrangements. We demonstrate 3D imaging of fluorescent beads and live cells with $3 \mu \mathrm{m}$ depth resolution. This approach has a potential application for depth resolved imaging, targeted photo-activation and optogenetics of cells in suspension and has the added benefit of potentially being resilient to scattering or aberrating media (including in vivo), a topic of current interest to both the imaging and trapping communities [9, 13, 16, 17]. The experimental setup is shown in Figure 1 and consists of optical paths for illumination, imaging and trapping. All three 
modalities share the same objective and thus the approach is compatible with a standard microscope. Temporal focusing (TF) of femtosecond laser pulses (center wavelength $795 \mathrm{~nm}$, repetition rate $80 \mathrm{MHz}$ ) is used for the illumination of the sample. The femtosecond laser pulses are derived from a Ti:Sapphire oscillator (KMLabs SWIFT-10) and temporally shaped (Biophotonic solutions MIIPS) in order to compensate for dispersion and deliver $20 \mathrm{fs}$ pulses at the sample plane. Temporal focusing is achieved via spectral dispersion of the incoming laser pulses by a transmission grating (300 lines $/ \mathrm{mm}$ ) with subsequent recombination of all the wavelengths by the objective in the imaging plane. The TF setup is integrated into the Nikon inverted microscope (Eclipse-Ti) equipped with a water immersion objective (Nikon CFI Plan Apo VC 60x WI, NA=1.2) and an Andor Clara CCD camera. The total imaging illumination power at the sample was approximately $80 \mathrm{~mW}$.

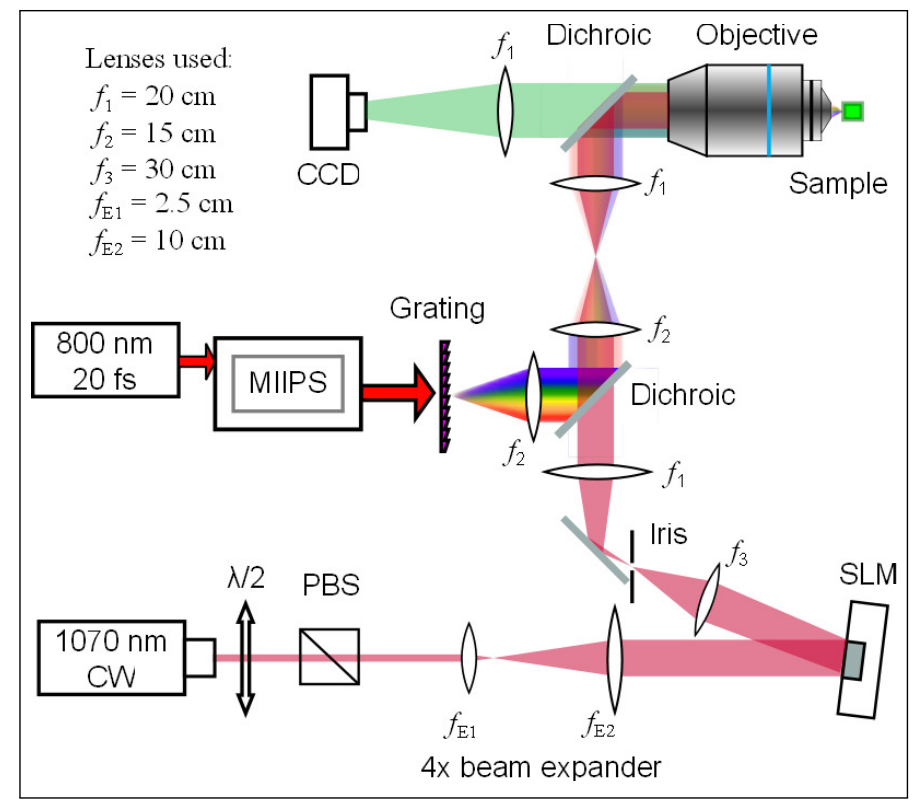

Fig. 1. Schematic diagram of the experimental setup. The trapping beam consists of a $1070 \mathrm{~nm} C W$ laser which passes through a half-waveplate $(\lambda / 2)$ and a polarizing beam splitter (PBS).The beam is then expanded onto the SLM, which is placed in a plane conjugate to the back aperture of the objective. The first order diffracted beam is separated from the other beam orders by an iris in the imaging plane. The illumination path consists of a $800 \mathrm{~nm}$ femtosecond laser and a pulse compression system (MIIPS). The pulses are dispersed using 300 lines/mm transmission grating and temporally focused by a 60x WI microscope objective. Two photon fluorescence is collected through the same objective and imaged onto an Andor Clara CCD camera.

The illumination profile and the depth resolution for TF have been characterized using a $350 \mathrm{~nm}$ spin coated sample of rhodamine-6G in PMMA. The illumination profile depends on the total magnification of the system and on the beam size at the grating position. The transverse profile of the illumination beam has a shape of an asymmetric Gaussian with FWHMs of $36 \mu \mathrm{m}$ and $22 \mu \mathrm{m}$ respectively. This defines the effective illumination area of the $60 x$ objective in our setup. The TF images were normalized by the illumination intensity profile. The illumination area can be varied by modifying the laser beam size at the grating position. The depth response function has FWHM of about $3 \mu \mathrm{m}$ when integrated across the whole illumination area. Figure 2 shows the two-photon fluorescence signal, produced by a $350 \mathrm{~nm}$ thin layer of rhodamine 6G, integrated over the $25 \times 40 \mu \mathrm{m}^{2}$ area. The depth resolution of our TF setup is comparable to the previously reported ones where a similar configuration with a linear grating has been used $[3,9,12]$.

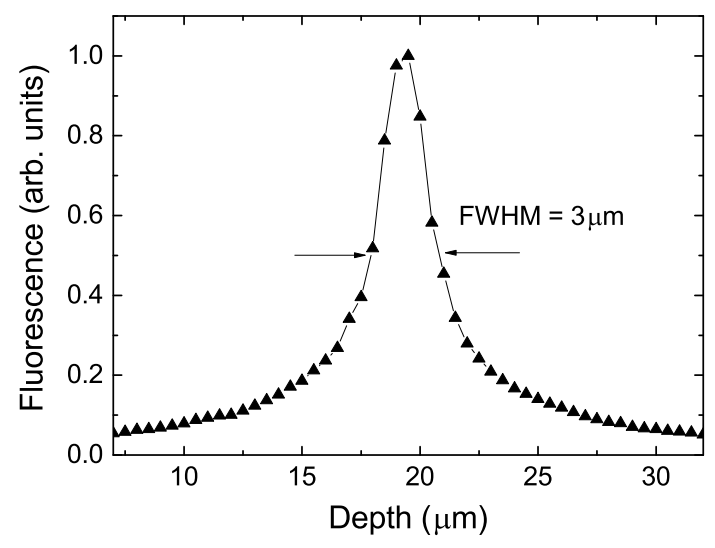

Fig. 2. Two-photon fluorescence from a $350 \mathrm{~nm}$ thin layer of fluorescent dye (Rhodamine 6G in PMMA) as a function of illumination depth. The fluorescent signal was integrated over the central part of the whole illumination area. The full width at half maximum was estimated to be around $3 \mu \mathrm{m}$ from the interpolation of the signal to $50 \%$ intensity level. The data set can be accessed at [23].

A 1064 nm CW (IPG) laser delivers the optical trapping beam. The laser beam is expanded onto the SLM (Hamamatsu LCOSSLM) which is placed in a conjugate plane to the back aperture of the objective. The first diffraction order of the trapping beam was selected by an iris placed in the image plane. The trapping beam is recombined with the illumination beam by a dichroic mirror. Both beams are delivered into the Nikon microscope and focused through the same microscope objective. Multiple traps were generated and controlled in 3D by shaping the trapping beam with appropriate SLM phase masks. The position of an individual trap was controlled by applying linear phases for the in plane motion and employing quadratic phase for the axial displacement [24]. The relation between the SLM phases and the trap position has been calibrated in three dimensions. The trap focus displacement was linear with respect to the SLM phases for all three axes. Multiple traps were generated and controlled by assigning different SLM pixels to different traps and controlling the phases of the corresponding set of pixels. Interference between the traps has been minimized by random pixel-to-trap assignment. During the experiment the laser power was adjusted to deliver about $50 \mathrm{~mW}$ per trap which is sufficiently low to maintain viability of biological samples for extended periods of time [25]. The performance of the setup was tested by imaging two $2 \mu \mathrm{m}$ diameter green fluorescent polymer microspheres trapped in water. The position of each sphere was controlled independently via SLM masks while the TF plane remained fixed. Figure 3 shows two-photon fluorescent temporal focusing (TPF-TF) imaging of trapped microspheres. The spheres were separated by $6 \mu \mathrm{m}$ in $x$ and $y$ directions and by $3 \mu \mathrm{m}$ in $z$ direc- 
tion (depth). Figure 3(a) shows bright-field image of the spheres where Figure 3(b) shows corresponding TPF-TF image. In order to obtain a 3D image both spheres were moved synchronously in the $z$ direction with a step of $500 \mathrm{~nm}$. Fluorescent image was acquired at each $z$ step. Figure 3(c) shows a rendered 3D image of the spheres which consists of 40 individual 2D slices. TPF-TF produces slightly elongated image of the spheres $(3.5 \mu \mathrm{m}$ instead of $2 \mu \mathrm{m}$ ) in the $z$ dimension which is consistent with our $3 \mu \mathrm{m}$ depth resolution.

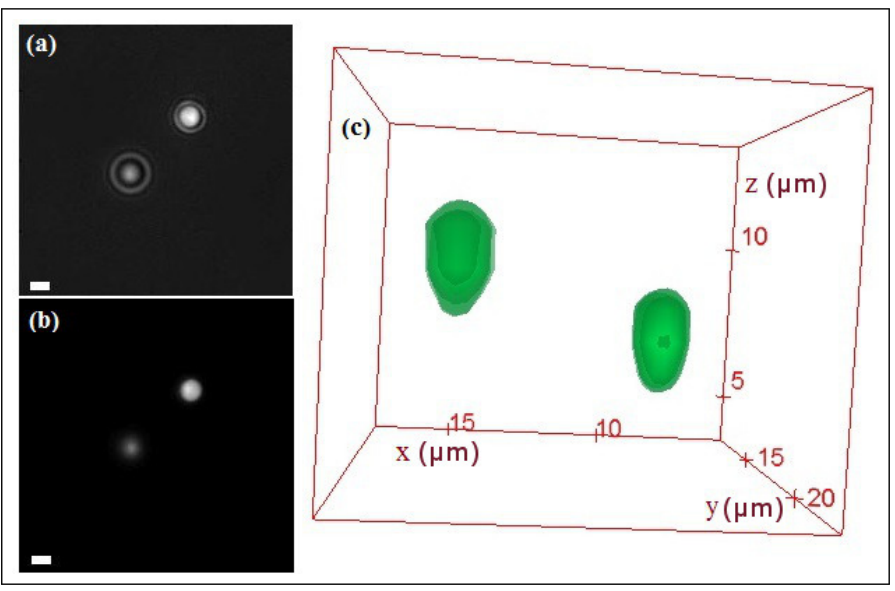

Fig. 3. Imaging of trapped beads separated by $6 \mu \mathrm{m}$ in $\mathrm{x}$ and y directions and by $3 \mu \mathrm{m}$ in depth. (a) Bright-field image and (b) corresponding TPF-TF image. The scale bar corresponds to $2 \mu \mathrm{m}$. (c) 3D rendered image reconstructed from 2D slices. The units on the axes correspond to $\mu \mathrm{m}$. The beads have been moved in the $z$ direction synchronously with a step of $500 \mathrm{~nm}$ by modifying the traps positions. The outer contour of each bead has been defined at $50 \%$ of maximum signal intensity level. The data set can be accessed at [23].

The TPF-TF 3D sectioning has also been applied to live HL60 cells stained with calcein-AM. Three cells were trapped and positioned with a $12 \mu \mathrm{m}$ separation in each of the $x$ and $y$ directions and $5 \mu \mathrm{m}$ in $z$ (depth). A bright-field (BF) image of the trapped cells is shown in Figure 4(a) and a corresponding TPF-TF image is presented in Figure 4(b). The dye is distributed within the cell, producing reasonably homogeneous fluorescence intensity across the cell body. The illumination can be directed to a different cell position by moving the cells with the SLM. The full depth scan of three trapped cells is shown in Figure 4(c). Although, all three cells are overlapped in the BF image, the TPF-TF images clearly show that only two cells are in physical contact while the third one is fully separated. The isolated cell is approximately $10 \mu \mathrm{m}$ in radius, where the other two cells are about $10 \mu \mathrm{m}$ to $20 \mu \mathrm{m}$ in size. The estimated illumination laser flux of $10^{4} \mathrm{~W} / \mathrm{cm}^{2}$ is sufficiently low, minimising phototoxicity and allowing the cells to remain viable for an extended period of time $[25,26]$.

The illumination area can be easily adjusted by changing the beam size at the grating or changing the objective magnification. Two-photon photo-activation is usually a photon intensive process. Therefore, the maximum illumination area can often be limited by the available laser power due to reduction in optical power per unit area for large illumination regions. To further improve the setup an independent control of TF illumination depth can be achieved by applying group velocity dispersion (GVD) to the illumination pulse [27]. The resolution of the TF setup

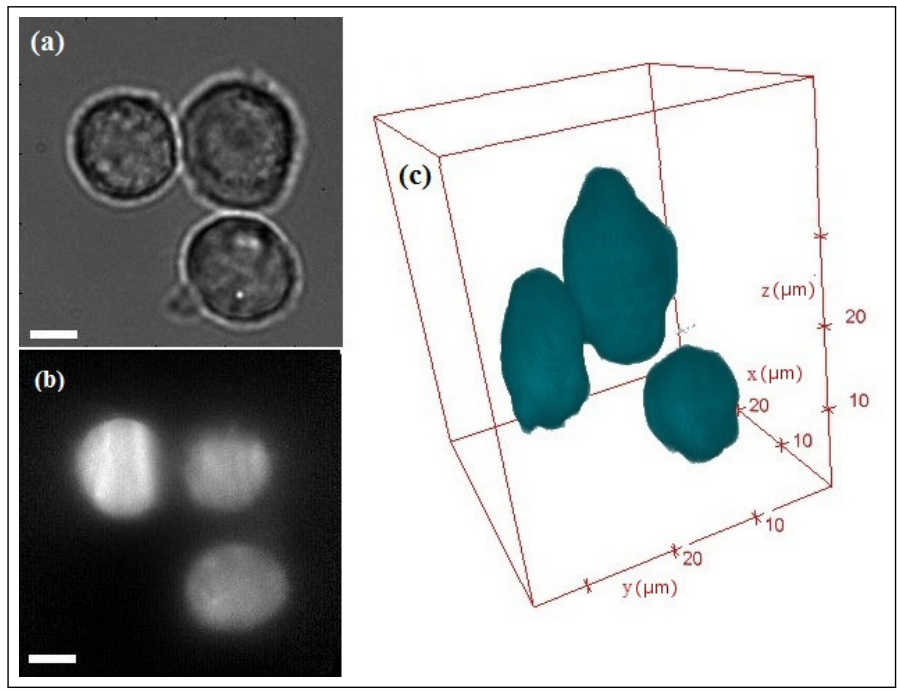

Fig. 4. TPF-TF imaging of trapped HL60 cells stained with calcein-AM. The cells are separated by $12 \mu \mathrm{m}$ in the transverse plane and by $5 \mu \mathrm{m}$ in the axial position. (a) Bright field image of three HL60 cells and (b) its corresponding TFF-TF image. The scale bar corresponds to $5 \mu \mathrm{m}$. (c) 3D image of trapped HL60 cells obtained by moving the axial position of the traps in steps of $500 \mathrm{~nm}$. The units on the axes correspond to $\mu \mathrm{m}$. The outer contours of the cells have been defined at $30 \%$ of maximum fluorescence intensity level. A total of 100 slices $(x, y$-plane images) were acquired with an integration time of one second each. The data set can be accessed at [23].

can be further improved by utilizing the full back focal aperture of the objective [11] and by using patterned illumination techniques $[8,12]$. This combination of holographic optical tweezers with temporal focusing allows for the simultaneous and independent positional control of a given cell or microparticle and depth-resolved wide-field illumination. As an example, it may be used for more advanced studies combined with the trapping of blood cells in vivo within an artery of a living animal [17] to study cells with 3D trapping and TF imaging through a single objective. The technique can be also applied to targeted photoactivation drug release in an artery where the optical trapping can be used as a precise delivery mechanism and TF can initiate the chemical release with a 3D precision. Other possible experiments using TF include monitoring of cellular Ca levels whilst under the influence of external objects optically positioned and manipulated near the cell membrane.

Standard multiphoton excitation techniques usually require time-consuming or computationally intensive wavefront corrections when used deep inside scattering media [28, 29]. The approach of TF on the other hand, can be applied beyond a single scattering length without any aberration corrections while preserving its spatial-temporal profile [5,9] yielding new opportunities. Since both the trapping and the TF techniques can be applied inside scattering media, they have the potential for targeted photo-activation of trapped objects in challenging biophotonics environments.

Funding. The UK Engineering and Physical Sciences Research Council (grant numbers: EP/M000869/1 and $\mathrm{EP} / \mathrm{J} 01771 \mathrm{X} / 1)$.

Acknowledgments. The authors thank Alison McDonald for providing the HL60 cells, and Mingzhou Chen for guidance with 
regard to the SLM. We also thank Nils M. Kronenberg and Guy Whitworth for help with preparing spin coated samples. KD acknowledges a Royal Society Leverhulme Trust Senior Fellowship.

\section{REFERENCES}

1. S. K. Mohanty, R. K. Reinscheid, X. Liu, N. Okamura, T. B. Krasieva, and M. W. Berns, Biophysical Journal 95, 3916 (2008).

2. D. Oron, E. Papagiakoumou, F. Anselmi, and V. Emiliani, vol. 196 of Progress in Brain Research, T. Knoepfel and E. S. Boyden, eds. (Elsevier, 2012), pp. $119-143$

3. D. Oron, E. Tal, and Y. Silberberg, Opt. Express 13, 1468 (2005).

4. G. Zhu, J. van Howe, M. Durst, W. Zipfel, and C. Xu, Opt. Express 13, 2153 (2005).

5. E. Papagiakoumou, F. Anselmi, A. Begue, V. de Sars, J. Gluckstad, E. Y. Isacoff, and V. Emiliani, Nat Meth 7, 848 (2010).

6. A. Vaziri, J. Tang, H. Shroff, and C. V. Shank, Proceedings of the National Academy of Sciences 105, 20221 (2008).

7. E. Block, M. Greco, D. Vitek, O. Masihzadeh, D. A. Ammar, M. Y. Kahook, N. Mandava, C. Durfee, and J. Squier, Biomed. Opt. Express 4, 831 (2013).

8. E. Y. S. Yew, H. Choi, D. Kim, and P. T. C. So, Proc. SPIE 7903, 790310 (2011).

9. E. Papagiakoumou, A. Begue, B. Leshem, O. Schwartz, B. M. Stell, J. Bradley, D. Oron, and V. Emiliani, Nat Photon 7, 274 (2013).

10. D. Kim and P. T. C. So, Opt. Lett. 35, 1602 (2010).

11. A. Vaziri and C. V. Shank, Opt. Express 18, 19645 (2010).

12. L.-C. Cheng, C.-H. Lien, Y. D. Sie, Y. Y. Hu, C.-Y. Lin, F.-C. Chien, C. Xu, C. Y. Dong, and S.-J. Chen, Biomed. Opt. Express 5, 2526 (2014).

13. T. Cizmar, M. Mazilu, and K. Dholakia, Nat Photon 4, 388 (2010).

14. A. Ashkin and J. Dziedzic, Science 235, 1517 (1987).

15. M. P. MacDonald, G. C. Spalding, and K. Dholakia, Nature 426, 421 (2003).

16. K. Dholakia, M. P. MacDonald, P. Zemánek, and T. Čižmár, vol. 82 of Methods in Cell Biology, M. W. Berns and K. O. Greulich, eds. (Academic Press, 2007), pp. 467.

17. M.-C. Zhong, X.-B. Wei, J.-H. Zhou, Z.-Q. Wang, and Y.-M. Li, Nat Commun 4, 1768 (2013).

18. M. Habaza, B. Gilboa, Y. Roichman, and N. T. Shaked, Opt. Lett. 40, $1881(2015)$

19. M. Yevnin, D. Kasimov, Y. Gluckman, Y. Ebenstein, and Y. Roichman, Biomed. Opt. Express 4, 2087 (2013).

20. A. Curran, S. Tuohy, D. G. A. L. Aarts, M. J. Booth, T. Wilson, and R. P. A. Dullens, Optica 1, 223 (2014).

21. K. Kim, J. Yoon, and Y. Park,Optica 2, 343 (2015).

22. A. Jesacher, S. Bernet, and M. Ritsch-Marte, Opt. Lett. 39, 5337-5340 (2014).

23. R. Spesyvtsev, H. A. Rendall, and K. Dholakia, http://dx.doi.org/10.17630/882664fc-9d26-4335-a013-81339d6962cd (2015).

24. J. Liesener, M. Reicherter, T. Haist, and H. Tiziani, Optics Communications 185, 77 (2000).

25. M. Goksör, J. Enger, and D. Hanstorp, Appl. Opt. 43, 4831 (2004).

26. J. M. Squirrell, D. L. Wokosin, J. G. White, and B. D. Bavister, Nat Biotech 17, 763 (1999).

27. B. Leshem, O. Hernandez, E. Papagiakoumou, V. Emiliani, and D. Oron, Opt. Express 22, 7087 (2014).

28. J. Yoon, M. Lee, K. Lee, N. Kim, J. M. Kim, J. Park, C. Choi, W. D. Heo, and Y. Park, http://arxiv.org/abs/1502.04826 (2015).

29. J.-H. Park, W. Sun, and M. Cui, Proceedings of the National Academy of Sciences 112, 9236 (2015).

\section{REFERENCES}

1. S. K. Mohanty, R. K. Reinscheid, X. Liu, N. Okamura, T. B. Krasieva, and M. W. Berns, "In-depth activation of channelrhodopsin 2-sensitized excitable cells with high spatial resolution using two-photon excitation with a near-infrared laser microbeam," Biophysical Journal 95, 3916 3926 (2008).

2. D. Oron, E. Papagiakoumou, F. Anselmi, and V. Emiliani, "Chapter 7 two-photon optogenetics," in "Optogenetics: Tools for Controlling and Monitoring Neuronal Activity,", vol. 196 of Progress in Brain Research, T. Knoepfel and E. S. Boyden, eds. (Elsevier, 2012), pp. $119-143$.

3. D. Oron, E. Tal, and Y. Silberberg, "Scanningless depth-resolved microscopy," Opt. Express 13, 1468-1476 (2005).

4. G. Zhu, J. van Howe, M. Durst, W. Zipfel, and C. Xu, "Simultaneous spatial and temporal focusing of femtosecond pulses," Opt. Express 13, 2153-2159 (2005).

5. E. Papagiakoumou, F. Anselmi, A. Begue, V. de Sars, J. Gluckstad, E. Y. Isacoff, and V. Emiliani, "Scanless two-photon excitation of channelrhodopsin-2," Nat Meth 7, 848-854 (2010).

6. A. Vaziri, J. Tang, H. Shroff, and C. V. Shank, "Multilayer threedimensional super resolution imaging of thick biological samples," Proceedings of the National Academy of Sciences 105, 20221-20226 (2008).

7. E. Block, M. Greco, D. Vitek, O. Masihzadeh, D. A. Ammar, M. Y. Kahook, N. Mandava, C. Durfee, and J. Squier, "Simultaneous spatial and temporal focusing for tissue ablation," Biomed. Opt. Express 4, 831-841 (2013).

8. E. Y. S. Yew, H. Choi, D. Kim, and P. T. C. So, "Wide-field two-photon microscopy with temporal focusing and hilo background rejection," Proc. SPIE 7903, 79031O-6 (2011).

9. E. Papagiakoumou, A. Begue, B. Leshem, O. Schwartz, B. M. Stell, J. Bradley, D. Oron, and V. Emiliani, "Functional patterned multiphoton excitation deep inside scattering tissue," Nat Photon 7, 274-278 (2013).

10. D. Kim and P. T. C. So, "High-throughput three-dimensional lithographic microfabrication," Opt. Lett. 35, 1602-1604 (2010).

11. A. Vaziri and C. V. Shank, "Ultrafast widefield optical sectioning microscopy by multifocal temporal focusing," Opt. Express 18, 1964519655 (2010).

12. L.-C. Cheng, C.-H. Lien, Y. D. Sie, Y. Y. Hu, C.-Y. Lin, F.-C. Chien, C. Xu, C. Y. Dong, and S.-J. Chen, "Nonlinear structured-illumination enhanced temporal focusing multiphoton excitation microscopy with a digital micromirror device," Biomed. Opt. Express 5, 2526-2536 (2014).

13. T. Cizmar, M. Mazilu, and K. Dholakia, "In situ wavefront correction and its application to micromanipulation," Nat Photon 4, 388-394 (2010).

14. A. Ashkin and J. Dziedzic, "Optical trapping and manipulation of viruses and bacteria," Science 235, 1517-1520 (1987)

15. M. P. MacDonald, G. C. Spalding, and K. Dholakia, "Microfluidic sorting in an optical lattice," Nature 426, 421-424 (2003).

16. K. Dholakia, M. P. MacDonald, P. Zemánek, and T. Čižmár, "Cellular and colloidal separation using optical forces," in "Laser Manipulation of Cells and Tissues,", vol. 82 of Methods in Cell Biology, M. W. Berns and K. O. Greulich, eds. (Academic Press, 2007), pp. $467-495$.

17. M.-C. Zhong, X.-B. Wei, J.-H. Zhou, Z.-Q. Wang, and Y.-M. Li, "Trapping red blood cells in living animals using optical tweezers," Nat Commun 4, 1768 (2013).

18. M. Habaza, B. Gilboa, Y. Roichman, and N. T. Shaked, "Tomographic phase microscopy with $180^{\circ}$ rotation of live cells in suspension by holographic optical tweezers," Opt. Lett. 40, 1881-1884 (2015).

19. M. Yevnin, D. Kasimov, Y. Gluckman, Y. Ebenstein, and Y. Roichman, "Independent and simultaneous three-dimensional optical trapping and imaging," Biomed. Opt. Express 4, 2087-2094 (2013).

20. A. Curran, S. Tuohy, D. G. A. L. Aarts, M. J. Booth, T. Wilson, and R. P. A. Dullens, "Decoupled and simultaneous three-dimensional imaging and optical manipulation through a single objective," Optica 1, 223-226 (2014).

21. K. Kim, J. Yoon, and Y. Park, "Simultaneous 3D visualization and position tracking of optically trapped particles using optical diffraction tomography," Optica 2, 343-346 (2015).

22. A. Jesacher, S. Bernet, and M. Ritsch-Marte, "Combined holographic optical trapping and optical image processing using a single diffractive pattern displayed on a spatial light modulator," Opt. Lett. 39, 5337-5340 (2014).

23. R. Spesyvtsev, H. A. Rendall, and K. Dholakia, "Data underpinning : 
Wide-field 3D optical imaging using temporal focusing for holographically trapped microparticles," http://dx.doi.org/10.17630/882664fc-9d264335-a013-81339d6962cd (2015).

24. J. Liesener, M. Reicherter, T. Haist, and H. Tiziani, "Multi-functional optical tweezers using computer-generated holograms," Optics Communications 185, $77-82$ (2000).

25. M. Goksör, J. Enger, and D. Hanstorp, "Optical manipulation in combination with multiphoton microscopy for single-cell studies," Appl. Opt. 43, 4831-4837 (2004).

26. J. M. Squirrell, D. L. Wokosin, J. G. White, and B. D. Bavister, "Longterm two-photon fluorescence imaging of mammalian embryos without compromising viability," Nat Biotech 17, 763-767 (1999).

27. B. Leshem, O. Hernandez, E. Papagiakoumou, V. Emiliani, and D. Oron, "When can temporally focused excitation be axially shifted by dispersion?" Opt. Express 22, 7087-7098 (2014).

28. J. Yoon, M. Lee, K. Lee, N. Kim, J. M. Kim, J. Park, C. Choi, W. D. Heo, and Y. Park, "Optogenetic signaling-pathway regulation through scattering skull using wavefront shaping," http://arxiv.org/abs/1502.04826 (2015).

29. J.-H. Park, W. Sun, and M. Cui, "High-resolution in vivo imaging of mouse brain through the intact skull," Proceedings of the National Academy of Sciences 112, 9236-9241 (2015). 